\title{
Deep VLT infrared observations of X-ray dim isolated neutron stars ${ }^{\star}$
}

\author{
G. Lo Curto ${ }^{1}$, R. P. Mignani ${ }^{2}$, R. Perna ${ }^{3}$, and G. L. Israel ${ }^{4}$ \\ 1 European Southern Observatory, Av. Alonso de Cordova 3107, Vitacura, Santiago, Chile \\ e-mail: glocurto@eso.org \\ 2 Mullard Space Science Laboratory, University College London, Holmbury St. Mary, Dorking, Surrey, RH5 6NT, UK \\ 3 JILA and Department of Astrophysical and Planetary Sciences, University of Colorado, 440 UCB, Boulder 80309, USA \\ 4 INAF Astronomical Observatory of Rome, Via Frascati 33, 00040 Monte Porzio Catone, Italy
}

Received 27 March 2007 / Accepted 26 June 2007

\begin{abstract}
Context. X-ray observations have unveiled the existence of a family of radio-quiet Isolated Neutron Stars whose X-ray emission is purely thermal, and hence are dubbed X-ray dim isolated neutron stars (XDINSs). While optical observations have allowed thermal emission to be related to the neutron star cooling and the neutron star surface thermal map to be built, IR observations are critical to pinpoint a spectral turnover produced by an as yet unseen magnetospheric component, or the presence of a fallback disk. The detection of such a turnover can provide further evidence of a link between this class of isolated neutron stars and the magnetars, which show a distinctive spectral flattening in the IR.

Aims. We present the deepest IR observations reported to date of five XDINSs, which we use to constrain a spectral turnover in the IR and the presence of a fallback disk.

Methods. We used archived VLT observations of these neutron stars performed with the ISAAC instrument in the $H$-band (1.65 $\mu \mathrm{m})$ and the available fallback disk models.

Results. It was not possible to identify the IR counterpart down to limiting magnitudes $H \sim 21.5-22.9$ for any of our targets. Although these limits are the deepest ever obtained for neutron stars of this class, they are not deep enough to rule out the existence and the nature of a possible spectral flattening in the IR. We also derive, using disk models, the upper limits on the mass inflow rate in a fallback disk. We find the existence of a putative fallback disk consistent (although not confirmed) with our observations.
\end{abstract}

Key words. accretion, accretion disks - techniques: photometric - astronomical data bases: miscellaneous - stars: neutron infrared: stars

\section{Introduction}

Observations performed at $\gamma$-ray and X-ray energies have unveiled the existence of peculiar classes of Isolated Neutron Stars (INSs) which stand apart from the family of more classical radio pulsars as they are, with only one confirmed exception (Camilo et al. 2006), radio-quiet. The soft gamma-ray repeaters (SGRs) and the anomalous X-ray pulsars (AXPs) are believed to be magnetars, i.e. young neutron stars with long spin periods (4-11 s) and hyper-strong magnetic fields $\left(B \sim 10^{14}-10^{15} \mathrm{G}\right.$, see Woods \& Thompson 2006). The magnetar model (Duncan \& Thompson 1992) would explain several observational properties of SGRs and AXPs, including the period, the spin down rate and their luminosity, in excess of what is expected from a standard pulsar spin down, see Woods \& Thompson (2006) for a recent review. Other radio-quiet INSs are the dim X-ray sources detected by ROSAT, hereafter X-ray Dim INSs or XDINSs, see Haberl (2007) for a review.

The XDINSs are characterized by steady emission patterns in the soft X-rays regime, where the spectra can be described by black-bodies with $40 \leq K T \leq 100 \mathrm{eV}$ and by high $\mathrm{X}$-ray to optical flux ratio $\left(\log \left(f_{\mathrm{X}} / f_{\text {opt }}\right) \approx 4-6\right)$. The XDINSs soft X-ray

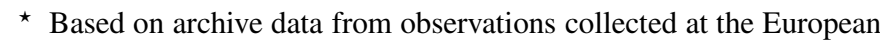
Southern Observatory, Paranal, Chile under programs IDs 071.C0189(A), 072.C-0051(A) and 074.C-0596(A). emission is likely powered by the cooling of the neutron star and it is pulsed with periods in the 3-11 s range.

According to cooling models (Page et al. 2006), the inferred surface temperatures imply that XDINSs ages are in the range of $\sim 10^{5}-10^{6}$ years which, in the case of RX J1856.6-3754 and RX J0720.4-3125, is compatible with their origin being in the Upper Scorpio and in the Trumpler 10 OB associations respectively, once their present position and proper motion are taken into account (Kaplan et al. 2007).

There is evidence which suggest that magnetars and XDINSs may be linked at some level. They have similar X-ray periods, and for four XDINSs, RX J1308.6+2127 (Haberl et al. 2003), RX J1605.3+3249 (van Kerkwijk et al. 2004), RX J0720.4-3125 (Haberl et al. 2004) and RX J2143.0+0654 (Zane et al. 2005) the observed X-ray absorption features, due either to proton cyclotron resonance or to atomic transitions, yield magnetic fields $B \sim 6-7 \times 10^{13}-1.0 \times 10^{14}$ G. For RX J0720.4-3125 and RX J1308.6+2127 the measured period derivatives $\dot{P} \sim 7 \times 10^{-14} \mathrm{~s}$ $\mathrm{s}^{-1}$ and $\dot{P} \sim 11 \times 10^{-14} \mathrm{~s} \mathrm{~s}^{-1}$ (Kaplan \& van Kerkwijk 2005a,b) imply, assuming magnetic dipole spin down, that $B \sim 3 \times 10^{13} \mathrm{G}$, i.e. consistent with the values derived from X-ray spectroscopy. In addition, for the measured parallactic distance of $360 \mathrm{pc}$ (Kaplan et al. 2007) the X-ray luminosity of RX J0720.4-3125 turns out to be larger than its inferred spin down energy, a characteristic which makes it similar to the magnetars which have an X-ray luminosity at least 2 orders of magnitudes higher with 
Table 1. Summary of the ISAAC observations of our targets. Column 2 gives the observing dates(s), Cols. 3 to 5 give the total integration time per observation, the average seeing and airmass. Column 6 gives the sky conditions.

\begin{tabular}{cccccl}
\hline \hline Target & $\begin{array}{c}\text { Date } \\
\text { dd-mm-yyyy }\end{array}$ & $\begin{array}{c}\text { Exp. time } \\
(\mathrm{s})\end{array}$ & $\begin{array}{c}\text { Seeing } \\
\text { (arcsec) }\end{array}$ & Airmass & Sky \\
\hline RX J0420.0-5022 & $14-11-2003$ & 1980 & 0.8 & 1.4 & clear \\
& $11-01-2004$ & 1980 & 0.6 & 1.3 & clouds \\
& $14-01-2004$ & 1980 & 1.1 & 1.7 & cyrrus \\
RX J0720.4-3125 & $05-12-2003$ & 1980 & 0.8 & 1.0 & photometric \\
& $09-01-2004$ & 1980 & 0.6 & 1.1 & cyrrus \\
& $12-01-2004$ & 1980 & 0.7 & 1.0 & clear \\
RX J0806.4-4123 & $26-11-2004$ & 3960 & 0.8 & 1.0 & clear \\
& $22-12-2004$ & 1980 & 0.6 & 1.1 & clear \\
RX J1856.5-3754 & $23-05-2003$ & 2760 & 0.6 & 1.1 & cyrrus \\
RX J2143.0+0654 & $07-06-2003$ & 2580 & 0.5 & 1.2 & cyrrus \\
\hline
\end{tabular}

respect to their spin down energy. In the IR, magnetars have a peculiar flux distribution which flattens with respect to the extrapolation of the X-ray spectra, see e.g. Israel et al. (2005). This flattening could be due either to the contribution of emission from a fallback disk, or to a turnover in the magnetospheric emission from the magnetar. In turn, the emission from the fallback disk could be generated by mass transfer and viscous dissipation within the disk and/or by re-processing of the X-rays from the neutron star. Were the XDINSs to show a similar energy distribution as the magnetars, flattening their spectra in the IR, it would strengthen the link between these two classes of objects.

In this paper we report on recent IR observations of five XDINSs: RX J0420.0-5022, RX J0720.4-3125, RX J0806.44123, RX J1856.6-3754 and RX J2143.6+0654. The paper is set out as follows: observations, data reduction and results are described in Sect. 2, while the discussion is presented in Sect. 3.

\section{Observations, data reduction, and results}

XDINSs IR observations were conducted using the NIR spectraimager ISAAC (Moorwood et al. 1998) mounted at the First Unit Telescope (UT1) of the ESO Very Large Telescope (VLT) at the Paranal Observatory in Chile under program IDs 071.C0189(A), 072.C-0051(A) and 074.C-0596(A), PIs R. Neuhäuser and B. Posselt. The instrument configuration was set to the short wavelength (SW) camera, equipped with a Rockwell Hawaii $1024 \times 1024$ pixel $\mathrm{Hg}$ :Cd:Te array, which has a pixel size of 0 '. 148 and a field of view of $152 \times 152$ arcsec. All observations were performed through the $H$-band filter $(\lambda=1.65 \mu \mathrm{m} ; \Delta \lambda=$ $0.30 \mu \mathrm{m})$. To allow for subtraction of the variable IR sky background, each exposure was split in sequences of 14 to 33 short randomly dithered exposures with detector integration times (DIT) of $12 \mathrm{~s}$, and 5 repetitions along each point of the dithering pattern. Observations were performed between May 2003 and December 2004 and a summary is shown in Table 1. Science exposures were retrieved through the public ESO archive $^{1}$ together with the closest-in-time calibration files. The data were reduced using the ESO's eclipse package (Devillard 2001) ${ }^{2}$ for de-jitter and sky subtraction. Unfortunately, we could not find in the ESO archive IR standard stars observed sufficiently close in date and time to our targets. We thus used the $H$-band flux of a number of $2 M A S S$ stars identified in the frames as relative calibration sources. Since the photometric accuracy of $2 M A S S$ is as good as $\sim 0.1 \mathrm{mag}$, and since a direct on-the-frame

\footnotetext{
1 http://archive.eso.org/

2 http://www.eso.org/projects/aot/eclipse/eug/ eug/eug.html
}

Table 2. Coordinates and position uncertainties of the observed objects. The uncertainties refer to a $90 \%$ confidence level for the sources not detected in optical (RX J0420.0-5022, RX J0806.4-4123, RX $\mathrm{J} 2143.0+0654)$. The reference epochs for the positions are detailed in the referenced papers: 1: Haberl et al. (2004), 2: van Kerkwijk \& Kaplan (2006), 3: van Kerkwijk \& Kulkarni (2001), 4: Rea et al. (2007).

\begin{tabular}{cllll}
\hline \hline Target & RA & Dec & $\Delta r$ & Ref. \\
\hline RX J0420.0-5022 & 042001.95 & -502248.1 & $0 . ' 6$ & 1 \\
RX J0720.4-3125 & 072024.96 & -312550.1 & 0.2 & 2 \\
RX J0806.4-4123 & 080623.40 & -412230.9 & $0^{\prime} 6$ & 1 \\
RX J1856.5-3754 & 185635.62 & -375435.3 & 0.2 & 3 \\
RX J2143.0+0654 & 214303.38 & +065417.5 & $0^{\prime \prime} 6$ & 4 \\
\hline
\end{tabular}

photometric calibration is not affected by overnight sky background fluctuations, we conclude that the accuracy of our photometric calibration is fully satisfactory. We used all available 2MASS stars in each frame (between 3 and 7) for the relative photometric calibration. Also, the wavelength overlap of the $2 M A S S$ and the ISAAC $H$-band filters is almost complete and the introduced error is within 0.03 mag. Photometry on the 2MASS stars was performed via standard aperture photometry with the Skycat - GAIA package (Draper \& Berry 2001). Frame distortions (amounting up to 2 pixels and depending on the position of the source in the frame) were not corrected for, and introduce an error of up to $0.1 \mathrm{mag}$ on the photometry of the individual 2MASS stars, which largely dominates the statistical errors. We finally derive a global photometric accuracy of $\sim 0.1-$ $0.2 \mathrm{mag}$.

The astrometric solution for the stacked ISAAC images was computed using as a reference, the positions and coordinates of stars selected from the $2 M A S S$ catalogue, yielding an rms between $0 .{ }^{\prime} 09$ and 0.14 . To register the positions of our targets we used as reference the most precise coordinates available in the literature (see Table 2). For RX J0420.0-5022 and RX J0806.44123 we have used the X-ray coordinates obtained with Chandra (Haberl et al. 2004), for RX J0720.4-3125 and RX J1856.63754 we have the coordinates of the optical counterparts after correcting for the measured proper motions (van Kerkwijk \& Kaplan 2006; and van Kerkwijk \& Kulkarni 2001, respectively), while for RX J2143.0+0654 we used the coordinates obtained with XMM (Zane et al. 2005). The overall uncertainties on the targets position account for the errors on the sky coordinates (see Table 2), for the rms of the astrometric solution, for the absolute uncertainty of the 2 MASS coordinates $(\sim 0.2)$ and, for RX J0720.4-3125 and RX J1856.6-3754, for the errors on the proper motion propagation between the reference and the observation epochs. 


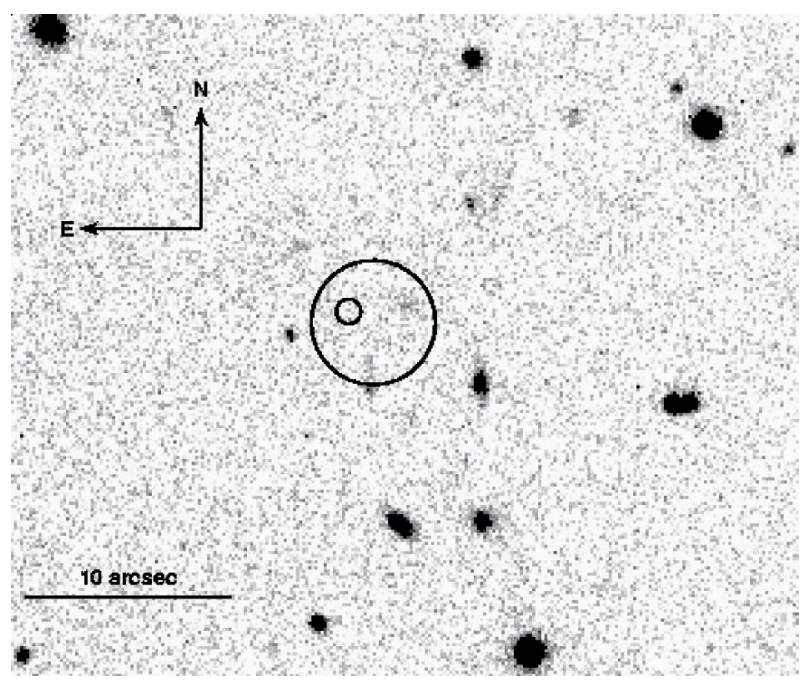

Fig. 1. ISAAC $H$-band images of the field of RX J2143.6+0654. Although two sources appear within the large XMM error circle, no source is visible within the smaller Chandra error circle. Moreover the two sources within the $X M M$ circle have large values of $B-V$, which are inconsistent with the nature of neutron stars emission, and have optical luminosity of a factor 10 higher than expected (see Mignani et al. 2007a, and the text).

Table 3. Summary of the $H$-band flux upper limits (expressed in magnitude) for the five XDINSs. Limiting magnitudes are computed for a $3 \sigma$ detection level. Columns 3 and 4 give the neutron star distance and the $N_{H}$, Col. 5 gives the interstellar extinction in the $H$-band, $A_{H}$, computed from the $N_{H}$ derived from the X-ray spectral fits using the relation of Paresce (1984) as done by, e.g. Posselt et al. (2007). We refer to Posselt et al. (2007) for the estimation of $N_{H}$, and the distances of RX J0420.0-502, RX J0806.4-4123 and RX J2143.0+0654. For objects with measured parallaxes, RX J0720.4-3125 and RX J1856.53754 , the distance measurements are taken from Kaplan et al. (2007) and from van Kerkwijk \& Kaplan (2006), respectively.

\begin{tabular}{l|c|lcc}
\hline \hline NS Name & $\begin{array}{c}H \\
\text { RX }\end{array}$ & $\begin{array}{l}d \\
\text { magnitude }\end{array}$ & $\begin{array}{c}N_{H} \\
10^{20} \mathrm{~cm}^{-2}\end{array}$ & $\begin{array}{c}A_{H} \\
\mathrm{mag}\end{array}$ \\
\hline J0420.0-5022 & $>21.7$ & $\approx 345$ & 1.6 & 0.016 \\
$\mathrm{~J} 0720.4-3125$ & $>22.7$ & $360_{-90}^{+170}$ & 1.2 & 0.012 \\
$\mathrm{~J} 0806.4-4123$ & $>22.9$ & $\approx 250$ & 1.0 & 0.010 \\
$\mathrm{~J} 1856.5-3754$ & $>21.5$ & $161_{-14}^{+18}$ & 0.7 & 0.007 \\
$\mathrm{~J} 2143.0+0654$ & $>22.1$ & $\approx 430$ & 2.4 & 0.024 \\
\hline
\end{tabular}

We do not detect any candidate IR counterpart for four of our sources, even extending the search area to 3 error circle radii (confirmed independently in Posselt 2007). In the case of RX J2143.0+0654, four possible counterparts were identified in Mignani et al. (2007a) based on their proximity with the XMM large error circle (Fig. 1), but they were all considered unlikely candidates due to their large $B-V>0.5$, to their optical brightness, a factor 10 higher than expected and, at least for two objects, to their apparent extension; the only point-like object within the XMM error circle in the IR frame has a low detection significance $(<5 \sigma)$. Very recently the Chandra position was published for this object (Rea et al. 2007). No sources are visible within the smaller Chandra error circle. Table 3 .

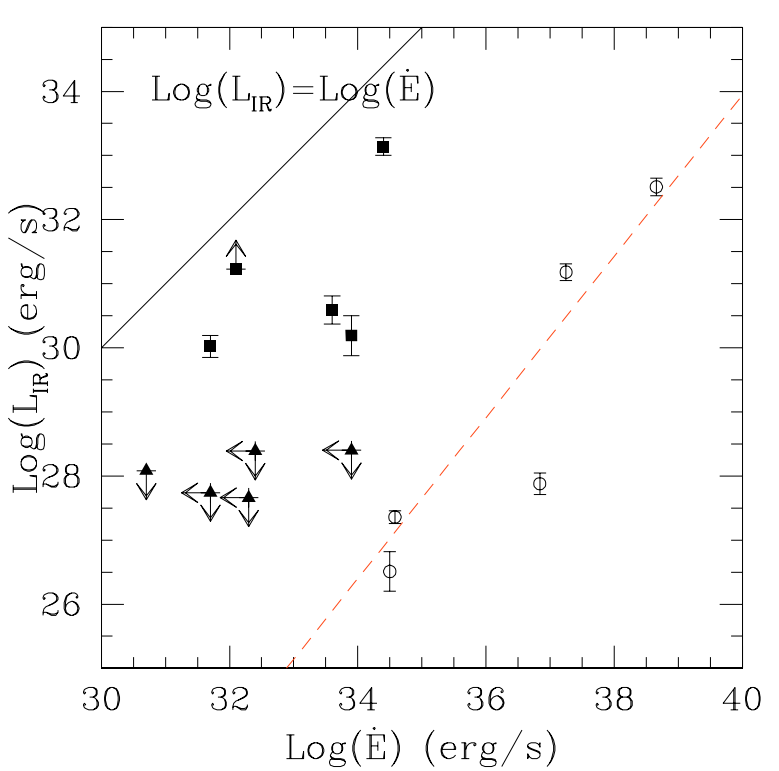

Fig. 2. IR luminosities of isolated NSs and derived XDINSs upper limits plotted as a function of the NSs rotational energy loss $\dot{E}$ (updated from Mignani et al. 2007b). Empty circles, filled squares and filled triangles indicate rotation-powered pulsars, magnetars, and XDINSs respectively. The dashed line corresponds to the linear fit for rotation-powered pulsars while the solid line shows the limit case $\log \left(L_{\mathrm{IR}}\right)=\log (\dot{E})$. For the rotation-powered pulsars and the magnetars the luminosity has been computed in the $K$-band, while for the XDINSs the luminosity upper limits refer to the $H$-band.

\section{Discussion}

We note that our derived IR flux upper limits are well above the extrapolation of the XDINSs' black body X-ray spectra, e.g. Haberl (2007), which predicts values of $H>25$. For RX J07203125 and RX J1856-3754, the only two XDINSs for which a reliable characterization of the optical flux distribution has been obtained (van Kerkwijk \& Kulkarni 2001; Motch et al. 2003), a hypothetical spectral flattening redward of the $R$-band would imply $H \leq 24$ and $H \leq 22$, respectively. The estimation is made assuming a constant flux normalized to the measured $R$-band magnitude of the targets. Comparable values can be expected for the other XDINSs in our sample assuming similar optical spectra and X-ray to optical flux normalizations.

We have used the measured $H$-band flux upper limits of the five XDINSs to derive their corresponding IR luminosities upper limits. Fluxes have been normalized either for the measured optical parallax distances (van Kerkwijk \& Kaplan 2006; Kaplan et al. 2007) or for the estimated distances presented in Posselt et al. (2007). The $H$-band interstellar extinction correction has been computed according to the relation of Fitzpatrick (1999) using as a reference the $A_{\mathrm{V}}$ inferred from the $N_{H}$ derived from the X-ray spectral fits in Posselt et al. (2007) and the relations of Predehl \& Schmitt (1995) and Paresce (1984). In both cases, the computed $H$-band interstellar extinction is significantly smaller than the global photometric uncertainty.

We have compared the XDINSs' IR luminosity upper limits with the measured IR luminosities of both rotation-powered pulsars and magnetars. For these objects, IR luminosities have been computed from the measured and the extrapolated $K$-band magnitudes (see Mignani et al. 2007b, for details) using the same procedure outlined above. Although the comparison between IR luminosities obtained in different bands is not formally correct, 
Table 4. Estimates of the upper limits of the accretion from a fallback disk. The luminosities reported in Col. 2 are referred to the distances in Col. 3 of Table 3. Columns 5 and 6 show the upper limits of the accretion rates (in units of $10^{-10} M_{\odot} y e^{-1}$ ) for the inner disk radius equal to the corotation radius and to the light cylinder radius, respectively. The X-ray luminosities are obtained from the measured XMM fluxes in the 0.1-2.4 keV energy band (Haberl 2004). References: (1) Haberl et al. (2004); (2) Zane et al. (2002); (3) Tiengo \& Mereghetti (2007); (4) Zane et al. (2005).

\begin{tabular}{lrrrccc}
\hline \hline Target & $\begin{array}{r}L_{\mathrm{X}} \\
10^{31} \mathrm{erg} \mathrm{s}^{-1}\end{array}$ & $\begin{array}{r}P \\
\mathrm{~s}\end{array}$ & $\begin{array}{c}\dot{P} \\
10^{-13} \mathrm{ss}^{-1}\end{array}$ & $\begin{array}{c}\dot{M}\left(R_{\text {in }}=R_{\text {cor }}\right) \\
10^{-10} M_{\odot} \text { year }^{-1}\end{array}$ & $\begin{array}{c}\dot{M}\left(R_{\text {in }}=R_{\mathrm{lc}}\right) \\
10^{-10} M_{\odot} \text { year }^{-1}\end{array}$ & Ref. \\
\hline RX J0420.0-5022 & 3.2 & 3.45 & $<92$ & 0.56 & 2.06 & 1 \\
RX J0720.4-3125 & 33.7 & 8.39 & 0.698 & - & 3.17 & 2 \\
RX J0806.4-4123 & 3.5 & 11.37 & $<18$ & 0.48 & 3.49 & 1 \\
RX J1856.5-3754 & 2.8 & 7.06 & $<19$ & 0.40 & 2.70 & 3 \\
RX J2143.0+0654 & 20.3 & 9.44 & $<60$ & 0.67 & 3.81 & 4 \\
\hline
\end{tabular}

the $H$ and $K$-bands are close enough in wavelengths that the error due to the unknown color correction is negligible with respect to the overall luminosity error budget. The comparison between different INS classes is shown in Fig. 2, where IR luminosities are plotted vs. the neutron stars' rotational energy loss $\dot{E}$ (assuming $I=10^{45} \mathrm{~g} \mathrm{~cm}^{2}$ ). As pointed out by Mignani et al. (2007b), the correlation between the IR luminosity of rotationpowered pulsars and $\dot{E}$ suggests that it is of magnetospheric origin, while the intrinsically higher IR luminosity of the magnetars is likely powered by the emission from a fallback disk and/or by the magnetic field decay. Since only upper limits on $L_{\mathrm{IR}}$ and $\dot{E}$ (with the only exception of RX J0720-3125) are available for the XDINSs, it is impossible to recognize a pattern in the diagram. XDINSs could either follow the same $L_{I R}$ vs $\dot{E}$ correlation of rotation-powered pulsars (or similar), and thus be magnetospheric emitters in the IR, or they might not follow any correlation at all, and thus be, e.g. mostly thermal emitters, as they are in the optical and X-rays. Alternatively, they could represent an intermediate class between the magnetars and the old rotationpowered pulsars, where all emission processes may coexist. In any case, their low IR emission clearly segregates them from magnetars. Whether this is due to an intrinsic difference between the two classes, or is simply due to the lower X-ray luminosity of the XDINSs which would reduce the contribution of the putative disk IR emission, or to a low mass inflow rate through such a disk (or both) is presently unclear.

Searches for fallback disks in different families of neutron stars were recently undertaken by several authors, e.g. Wang et al. (2007), Mignani et al. (2007b) on four compact central objects and on one high magnetic field radio pulsar respectively, both reporting non-detections. Instead Wang et al. (2006) successfully detected a flattening in the spectrum of the AXP $4 \mathrm{U} 0142+61$ from Keck ( $K$-band) and Spitzer observations, interpreting the data as a detection of a fallback disk of mass $\approx 10^{-5} M_{\odot}$ around the neutron star. The colors of the detected source are unusually red with respect to main sequence stars or giant stars. Fallback disks could also be found in principle around XDINSs (Perna et al. 2000; Michel \& Dessler 1981).

To set constraints on the presence of underlying fallback disks around the XDINSs in our sample, we used the derived $H$-band flux upper limits. Since these objects do not display radio emission, a hypothetical disk could extend well inside the light cylinder radius. We note that the detection of pulsed radio emission at very low frequencies $(42-112 \mathrm{MHz})$ was recently reported for RX J1308.6+2127 and RX J2143.0+0654 (Malofeev et al. 2007) but has not been yet independently confirmed. The inner radius of the disk is generally expected at the magnetospheric radius. Since this is dependent on the magnetic field and on the accretion rate of the disk which is not known a priori, this radius cannot be computed (Chatterjee et al. 2000). However, given the very low X-ray luminosity level of the XDINSs, we do not expect that a hypothetical fallback disk would accrete on the neutron star, which implies that the minimum value that the magnetospheric radius can take is the corotation radius. Since the corotation radius only depends on the neutron star period, it can be computed for all of our objects. The disk emission was computed using the models of Perna et al. (2000), which include both the contribution to the emission from viscous dissipation, as well as the contribution from reprocessing of the Xray flux. However, given the very low X-ray luminosity level of most XDINSs (see Table 4), we found that, for fluxes below (but close) to the IR limits, the contribution to the disk emission is generally dominated by the viscous dissipation part. Therefore, our observational limits translate into limits on the maximum possible mass inflow rate through the disk. The exception is represented by RX J0720.4-3125, which has the largest X-ray luminosity and for which the flux from a disk with an inner edge at the co-rotation radius would be instead dominated by the reprocessing of the X-ray emission from the neutron star. The expected disk IR emission would in this case be brighter than our derived upper limit, unless the disk inner radius is much larger than the co-rotation radius, or the disk has an inclination with respect to the line of sight which is higher than the assumed $60^{\circ}$.

The results are shown in Table 4 , where the maximum $\dot{M}$ compatible with the limits is reported. For each object, we derived the limits using two different values for the inner radius of the disk: $R_{\text {in }}=R_{\text {cor }}$ and $R_{\text {in }}=R_{\mathrm{lc}}$, where $R_{\text {cor }}$ is the corotation radius (the absolute minimum), and $R_{\mathrm{lc}}$ is the light cylinder radius (for reference). Clearly, when the radius is closer in (i.e. when $R_{\text {in }}=R_{\text {cor }}$ ), the limits on $\dot{M}$ are more stringent, due to the fact that there is a larger contribution of flux coming from the inner regions of the disk. This translates to an upper limit of the mass inflow though the disk of at least $\approx 0.4 \times 10^{-10} M_{\odot}$ year $^{-1}$.

\section{Conclusions}

We report on the deepest IR observations ever, of 5 of the 7 known XDINSs. None of the sources were detected in our data at the $3 \sigma$ level. Limiting $H$-band magnitudes are in the $\approx 21.5-22.9$ range. These limits do not allow us to either exclude or confirm a spectral flattening red-ward of the $R$-band as observed in some magnetars.

We have compared the derived IR luminosity upper limits with the measured values of other classes of isolated neutron stars. Interestingly, we found that the distribution of our targets in the $L_{\mathrm{IR}}-\dot{E}$ plane shows a separation between the XDINSs and the magnetars due to their lower (by at least two orders of magnitude) IR luminosity. Due to the indetermination we have 
on the IR flux and on $\dot{P}$ we can neither exclude nor confirm that XDINSs will follow a trend similar to the rotation-powered pulsars in the $L_{\mathrm{IR}}-\dot{E}$ plane, implying IR emission from the magnetosphere.

We investigated the possible existence of fallback disks around our targets and found that the contribution of the disk emission would be mostly dominated by viscous dissipation. Although we cannot confirm the existence of fallback disks, we have derived disk mass inflow rate upper limits which are consistent with the age and the disk mass estimates of our targets.

Multi-color deeper IR observations, both in the near IR and in the medium IR (Spitzer) bands, could better probe the possibility of spectral flattening in the IR and its origin.

Acknowledgements. R.P.M. and G.L.I. acknowledge the ESO/Chile Scientific Visitors Programme for supporting their stay at the ESO Santiago Offices (Vitacura) where most of this work was finalised. RP warmly thanks the ESO/Chile Scientific Visitors Programme for the hospitality during her visit. G.L.C. thanks the Rome observatory in Monte Porzio Catone for the hospitality.

\section{References}

Camilo, F., Ransom, S. M., Halpern, J. P., et al. 2006, Nature, 442, 892 Chatterjee, P., Hernquist, L., \& Narayan, R. 2000, ApJ, 534, 373 Devillard, N. 2001, in Astronomical Data Analysis Software and Systems X, ed.

F. R. Harnden, Jr., F. A. Primini, \& H. E. Payne, ASP Conf. Ser., 238, 525

Duncan, R. C., \& Thompson, C. 1992, ApJ, 392, L9

Fitzpatrick, E. L. 1999, PASP, 111, 63

Haberl, F. 2004, Adv. Space Res., 33, 638

Haberl, F. 2007, Ap\&SS, 73 [astro-ph/0609066]
Haberl, F., Schwope, A. D., Hambaryan, V., Hasinger, G., \& Motch, C. 2003, A\&A, 403, L19

Haberl, F., Motch, C., Zavlin, V. E., et al. 2004, A\&A, 424, 635

Israel, G., Covino, S., Mignani, R., et al. 2005, A\&A, 438, L1

Kaplan, D. L., \& van Kerkwijk, M. H. 2005a, ApJ, 628, L45

Kaplan, D. L., \& van Kerkwijk, M. H. 2005b, ApJ, 635, L65

Kaplan, D. L., van Kerkwijk, M. H., \& Anderson, J. 2007, Ap\&SS, in press [astro-ph/0703343]

Malofeev, V. M., Malov, O. I., \& Teplykh, D. A. 2007, Ap\&SS, 72

Michel, F. C., \& Dessler, A. J. 1981, ApJ, 251, 654

Mignani, R. P., Bagnulo, S., de Luca, A., et al. 2007a, Ap\&SS, 71 [astro-ph/0608025]

Mignani, R. P., Perna, R., Rea, N., et al. 2007b, A\&A, submitted

Moorwood, A., Cuby, J.-G., Biereichel, P., et al. 1998, The Messenger, 94, 7

Motch, C., Zavlin, V. E., \& Haberl, F. 2003, A\&A, 408, 323

Page, D., Geppert, U., \& Weber, F. 2006, Nucl. Phys. A, 777, 497

Paresce, F. 1984, AJ, 89, 1022

Perna, R., Hernquist, L., \& Narayan, R. 2000, ApJ, 541, 344

Posselt, B. 2007, Ph.D. Thesis,

http: //www.db-thueringen . de/servlets/DocumentServlet?id=8197

Posselt, B., Popov, S. B., Haberl, F., et al. 2007, Ap\&SS, 75

P.W. Draper, N. G., \& Berry, D. 2001, GAIA - Graphical Astronomy and Image Analysis Tool, sun/214.9 edn., Starlink

Rea, N., Torres, M. A. P., Jonker, P. G., et al. 2007, ArXiv e-prints, 705

Tiengo, A., \& Mereghetti, S. 2007, ApJ, 657, L101

van Kerkwijk, M. H., \& Kaplan, D. L. 2006, Ap\&SS, in press, [astro-ph/0607320]

van Kerkwijk, M. H., Kaplan, D. L., Durant, M., Kulkarni, S. R., \& Paerels, F. 2004, ApJ, 608, 432

van Kerkwijk, M. H., \& Kulkarni, S. R. 2001, A\&A, 378, 986

Wang, Z., Chakrabarty, D., \& Kaplan, D. L. 2006, Nature, 440, 772

Wang, Z., Kaplan, D. L., \& Chakrabarty, D. 2007, ApJ, 655, 261

Woods, P. M., \& Thompson, C. 2006, Soft gamma repeaters and anomalous

X-ray pulsars: magnetar candidates (Compact stellar X-ray sources), 547

Zane, S., Haberl, F., Cropper, M., et al. 2002, MNRAS, 334, 345

Zane, S., Cropper, M., Turolla, R., et al. 2005, ApJ, 627, 397 